\title{
Design about Simple Tester of Low Capacitance Based on MAX038 Zheng Liping ${ }^{1, a}$ \\ ${ }^{1}$ Photoelectric Engineering College of Yunnan Open University, Kunming, China, 650500 \\ azhengliping1209@163.com
}

Keywords: MAX038, test of low capacitance, TM4C123GH6PM, frequency measurement by equal precision

\begin{abstract}
Simple tester of low capacitance was designed based on MAX038 in this paper. The principle is that external capacitor of MAX038 as test capacitor to provide corresponding frequency signal output, and TM4C123GH6PM was selected to measure frequency by equal precision and calculate test capacitance. In order to improve the accuracy, data were piecewise fitted by the least square method, and comparison tests were between high accuracy capacitance tester and the simple tester to realize auto correction and show measurement results. The tester can detect $10 \mathrm{pF} \sim 1 \mu \mathrm{F}$ capacitor. Test results show that the tester is running stable, rapid measuring; accuracy is grade 1 .
\end{abstract}

\section{Introduction}

In this paper, through the research of how standard function signals are generated and the relationship between signal frequency and capacitance values, we designed this portable capacitor tester based on MAX038. In the design, we applied digital signal processing technique $[1,4]$ and the method of ratio correcting [5], making the tester faster and more accurate. It can be functioned not only as a general portable capacitance tester, but also as suitable subject for students, so that they can grow through practice, and be excellent after repeated renovation.

\section{The Hardware Components and Working Principle of Simple Tester of Low Capacitance}

The capacitance test system designed in this paper include power module, function signal generator module, zoom conditioning module, TM4C123GH6PM control system and display module, the systematic structure can be shown in Figure 1. Among them, the power supply module provides a function generator MAX038 $\pm 5 \mathrm{~V}$ power supply, while the use of low-dropout linear regulator LM1117 make the voltage drop from $5 \mathrm{~V}$ to $3.3 \mathrm{~V}$, and provide power for TM4C123GH6PM; MAX038 function generator can provide an accurate square wave signal, whose frequency is associated with the measured capacitance; after the conditioned square wave signal being amplified, its frequency can be estimated by TM4C123GH6PM control system, and then calculate the capacitance value of the measured capacitance, and finally display it on the LCD screen.

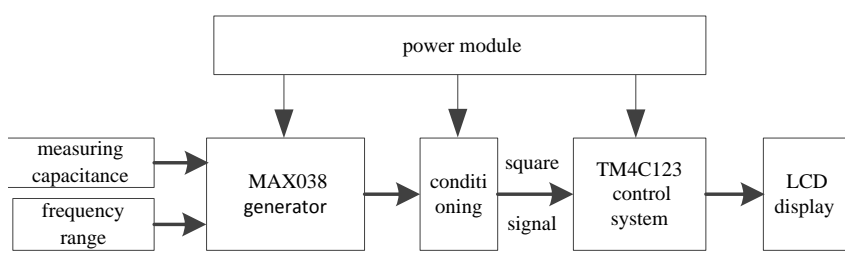

Fig.1 System principle block diagram

\section{Function Generator and Frequency Range Selection}

\section{(1) Function Generator MAX038}

The function signal generating module is to generate a precise signal, whose frequency is associated with the capacitor, by measuring the frequency of the signal we can figure out the value of the capacitor. The MAX038 adopted in this design is a low-distortion, high-frequency, 
high-precision monolithic integrated function generator, and with the help of a small number of external components it can produce accurate high-frequency triangle wave, sine wave, square wave, and its output frequency range is between $0.1 \mathrm{~Hz}$ and $20 \mathrm{MHz}$. The frequency of the output signal of MAX038 is decided by the input voltage UFADJ of modulation, the reference current IIN and external oscillation capacitor.

When $U_{\mathrm{FADJ}}=0 \mathrm{~V}$, link a resistor $R_{\mathrm{IN}}$ to the place between the IIN terminal and the output terminal of reference voltage, the outputted oscillation frequency is

$f_{0}=\frac{U_{\mathrm{REF}}}{R_{\mathrm{IN}} C_{\mathrm{F}}}$

Which, $C_{\mathrm{F}}$ is the measured capacitance that attached to the outside part of MAX038.

(2)Frequency Range Selection

In this test system, under the premise of enlarging the measurement value as large as possible, the external resistor $R_{\mathrm{IN}}$ should meet two conditions: (1) the output frequency of MAX038 should not be too low, otherwise the measurement accuracy of equal precision frequency measurement will be reduced; take output frequency of $50 \mathrm{~Hz}$ for example, the external resistor RIN should not exceed $5 \mathrm{~K} \Omega$. (2) Ensure that the output frequency does not exceed $20 \mathrm{MHz}$, you can try resistors with greater resistance, but you should also take into account the optimal operating current range of IIN

For the above factors in this test system, the three kinds of external resistance values divide the capacitance measurement into two positions: Take $R_{\mathrm{IN}}=12.5 \mathrm{~K} \Omega$ for $1 \mu \mathrm{F} \sim 1 \mathrm{nF}$ files; take $R_{\mathrm{IN}}=$ $25 \mathrm{~K} \Omega$ for $1000 \mathrm{pF} \sim 10 \mathrm{pF}$ files.

\section{Enlarged Conditioning Module}

After the output square wave signal of MAX038 being amplified and conditioned, the amplitude is now acceptable to TM4C123GH6PM. The module is connected to the same phase ratio AD8048 amplifier circuit, and in accordance with the output signal of 10pF capacitor of MAX038, amplify about $3 \mathrm{~V}$ to regulate the amplified circuit gain.

\section{TM4C123 Control Module}

The control system made up of TM4C123GH6PM microcontroller achieves two main functions: First, use equal precision frequency measurement method to measure MAX038 output square wave signal frequency; the second is the application of least squares fitting and alignment correction method, which can accurately calculate and display the measured capacitance value.

The waveform of precision frequency measurement can be shown in Figure 2. The inner part of TM4C123GH6PM generates a gate signal, and within the gate, counter A and counter B count respectively the measured signals(square wave frequency $f_{\mathrm{x}}$ ) and standard frequency signals (time scale frequency $f_{\mathrm{c}}$ ), assume that within a set time $\mathrm{T}$, the $f_{\mathrm{x}}$ number is $N_{\mathrm{A}}$, and $f_{\mathrm{c}}$ number is $N_{\mathbf{B}}$. Then we have:

$f_{\mathrm{x}}=\frac{f_{\mathrm{c}} N_{\mathrm{A}}}{N_{\mathrm{B}}}$

When the gate signal $T=1 \mathrm{~s}$, the counted number $N_{\mathrm{A}}$ of the measured signal is the frequency output value of the measured capacitance, namely,

$f_{\mathrm{x}}=N_{\mathrm{A}}$

Therefore, the actual circuit design eliminates the need for timing signal frequency $f_{\mathrm{c}}$ counter, because the square wave frequency can be obtained by counter A. And when TM4C123GH6PM put the estimated frequency value into the formula, the measured capacitance values can be obtained and displayed. 


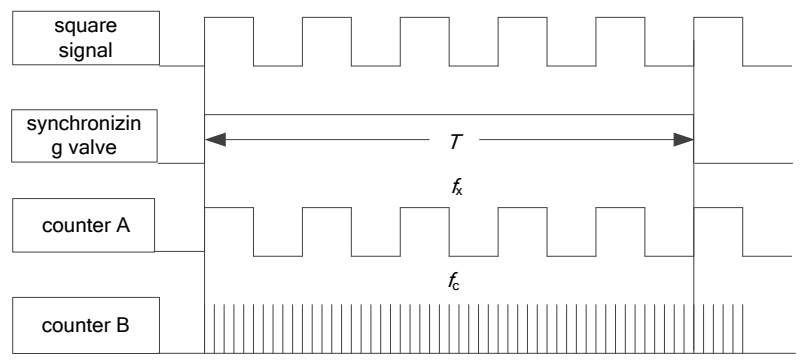

Fig.2 Oscillogram of frequency measurement by equal precision

\section{Design of Capacitor Tester Software}

System software design consists of two modules, namely the main function and timer interrupt function. The former mainly deals with microcontroller TM4C123GH6PM, which include configuration initialization, display control initialization and interrupt initialization; and calculate the signal frequency and capacitance values. While the latter is mainly used for timin, the flowcharts of the two can be shown in Figure 3.

When the main program detects the rising edge of the signal under test, clears the counter, at the same time interrupt the $1 \mathrm{~s}$ timer, then a 1 -second synchronous gate signal will be generated. Count the amplified square wave signals outputted by MAX038.

\section{Partitioned Imitation Under Least Square Method}

In order to improve the accuracy of the tester, use the least squares method [10] to deal with the two test capacitors, and make it form a fitted curve in accordance with the formula MATLABC $=$ $a / f+b$. The fitting formula parameter and the curve can be shown in in Figure 3 . Write the fitted formula into TM4C123GH6PM, you can estimate the capacitance value according to different stalls. And figure out its average value through repeated estimation to minimize the random error; use high-accuracy LCR meter to carry out the comparison test, and to correct system errors.
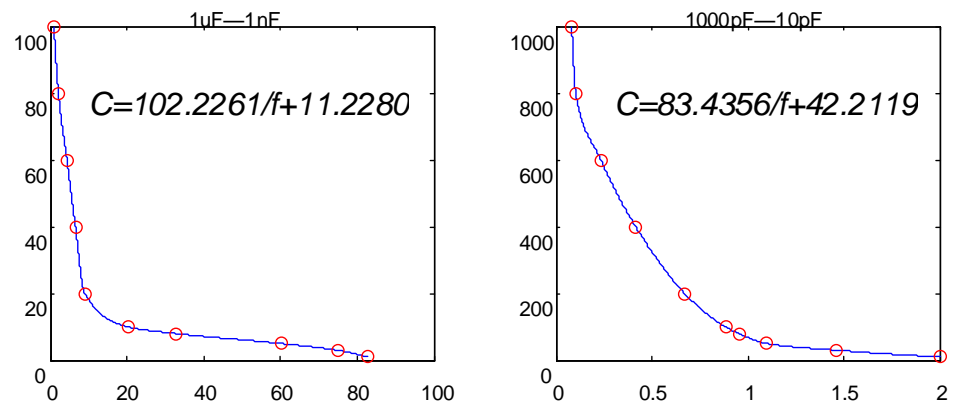

Fig.3 Piecewise fitted curves by least square method

\section{Test Results and Error Analysis}

\section{Test Principle}

Check the same test source with an instrument with a high level of accuracy and this tester to set the tester's test grades. Test schematic diagram is shown in Figure 4. 


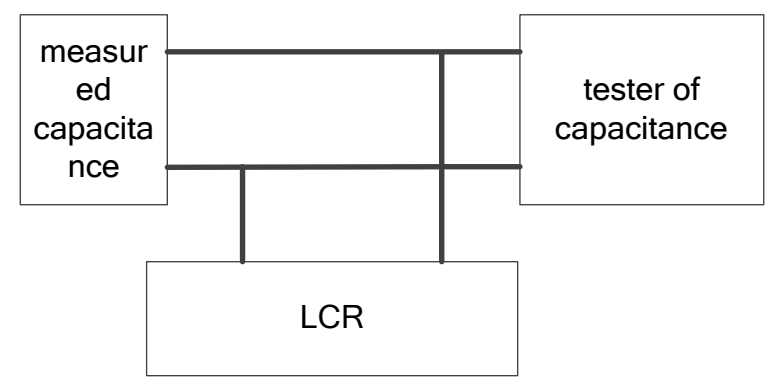

Fig.4 Measurement principle block diagram

\section{Testing Instrument}

Use TH2821A handheld LCR digital bridge as its test instrument. And its capacitance test range: $1 \mathrm{pF} \sim 9999 \mu \mathrm{F} ; 1 \mathrm{kHz}, 10 \mathrm{kHz}: 0.1 \mathrm{pF} \sim 999.9 \mu \mathrm{F} ;$ measuring accuracy $0.3 \%$.

\section{Measurement results}

Test data are shown in Table 1. As can be seen from the measurement results in the range of $10 \mathrm{pF} \sim 1 \mu \mathrm{F}$, the maximum error value references appear when the standard capacitance value stay at point $1.037 \mu \mathrm{F}$, namely, $0.87 \%$, and the measurement accuracy of this tester is grade one.

Tab.1 Measurement results

\begin{tabular}{lccccc}
\hline reference value & 1.037 & 0.104 & 0.0516 & 0.0099 & 0.0012 \\
\hline measured value & 1.046 & 0.105 & 0.0518 & 0.0010 & 0.0011 \\
reference value & 103.4 & 52.0 & 21.9 & 11.4 & 0.0019 \\
measured value & 102.5 & 52.5 & 22.1 & 11.3 & 0.1109 \\
\hline
\end{tabular}

\section{Conclusion}

The capacitive test based on MAX038 function signal generator regards the external capacitor of function signal generator as the measured capacitance, and it can produce corresponding frequency signal output; based on the measured frequency value of the external resistor, we can calculate the capacitance value. Use the binal least squares technique and alignment correction method to improve the accuracy of the test. The test results show that within the range of $10 \mathrm{pF} \sim 1 \mu \mathrm{F}$, test accuracy is better than grade one. The tester can measure the capacitance value in a fast and more accurate way, and can be used as a general portable small capacitance tester.

\section{Acknowledgement}

In this paper, the research was sponsored by the Study on the process of turning the germanium crystal in optical parts process, scientific research fund project of Yunnan Province of Education, 2011. Item No. 2011C161)

\section{References}

[1] Su Lliang. Design and Research of Portable Capacitance Tester [D]. Wuhan: Huazhong University of Science and Technology, 2007.

[2] Liu Jun, Li Zhi. Microcontroller-based High-Precision Inductance, Capacitance Tester [J] Research and Development, 2007,26 (6): 48-51.

[3] Shen Xiaogu. Using pulse counting method to measure capacitance MCU [J] Shanghai Institute of Technology, 2006,6 (4): 290-293. 
[4] Y. Geng, J. Chen, K. Pahlavan, Motion detection using RF signals for the first responder in emergency operations: A PHASER project, 2013 IEEE 24nd International Symposium on Personal Indoor and Mobile Radio Communications (PIMRC), London,Britain Sep. 2013

[5] Y. Geng, J. He, K. Pahlavan, Modeling the Effect of Human Body on TOA Based Indoor Human Tracking[J], International Journal of Wireless Information Networks 20(4), 306-317 